\section{Wenn Allergien durch den Magen gehen ...}

D ie Diagnostik der Nahrungsmittelallergien oder -intoleranzen gehört zu den schwierigsten Aufgaben des Allergologen. Durch umfassende Behandlung in der Laienpresse hat das Thema im Bewusstsein der Bevölkerung in den letzten Jahren einen bedeutenden Stellenwert eingenommen. So ist die Zeit gut gewählt für die zweite Auflage dieses Buches.

Die Definition der Nahrungsmittelallergie im Vergleich zur -intoleranz wird zu Beginn ausführlich dargelegt. Ebenso wird gezeigt, dass echte Allergien auf Nahrungsmittel äußerst selten vorkommen.

Bevor die Autoren auf die einzelnen Allergien eingehen und deren Diagnostik beschreiben, werden in zwei Kapiteln die Physiologie des Magen-DarmTraktes sowie die Pathogenese und Pathophysiologie eindrucksvoll und überschaubar dargelegt. Die immunologischen Voraussetzungen werden unter Berücksichtigung der neuesten wissen- schaftlichen Erkenntnisse verständlich gemacht.

Mit dieser Basis ist es auch dem in der Materie Unerfahrenen gut möglich, das recht komplexe Krankheitsbild und die daraus erwachsenden analytischen und therapeutischen Strategien nachzuvollziehen. Besonders hervorzuheben ist die ausführliche Besprechung der einzelnen Nahrungsmittel und deren Allergenität.

Bei der Behandlung von Nahrungsmittelallergien ist bekannt, dass die Karenz des auslösenden Allergens die beste und sicherste Therapie ist. Aber auch dem erfahrenen Allergologen sind sicher nicht immer alle Inhaltsstoffe bekannt und so sind das kleine Gewürzlexikon und der Diätplan ein hilfreicher Ratgeber.
Die Autoren haben mit diesem Buch ein Werk geschaffen, das die komplizierten Zusammenhänge bei Nahrungsmittelallergien und -intoleranzen in Wort, Zeichnungen und Tabellen optimal veranschaulicht. Es ist gleichermaßen geeignet als Lehrbuch und Nachschlagewerk und kann zur Verbesserung des Qualitätsstandards einer allergologischen Fachpraxis beitragen. Gleichzeitig ist es aber auch für den oft ersten Ansprechpartner der Patienten - den Hausarzt - bestens geeignet.

Dr. Bettina Hilka, Wiesbaden

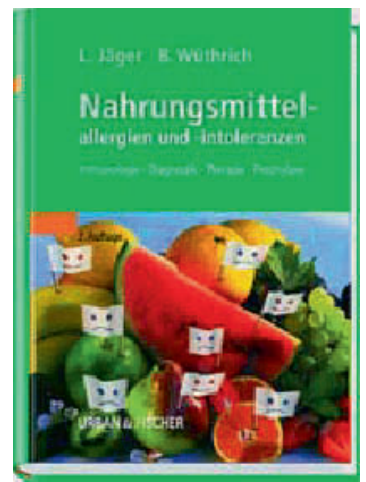

Jäger L, Wüthrich B.

Nahrungsmittelallergien und -intoleranzen. Immunologie - Diagnostik -Therapie - Prophylaxe. München - Jena: Urban \& Fischer, 2002.

59,95 E.

ISBN 3-437-21361-X

\section{Was essen bei Nahrungsmittelallergie?}

A n Ärzte, Betreuer und Betroffene gleichermaßen richtet sich dieses Taschenbuch zur Diät bei Nahrungsmittelallergien und -intoleranzen. Die Autoren geben umfassende Informationen zu den Überempfindlichkeitsreaktionen auf verschiedene Nahrungsmittelgruppen und -bestandteile in einer bemerkenswert klaren Gliederung. Besonders erwähnenswert ist, dass die Autoren auch Zusatzinformation - z. B. über mögliche Mangelernährungen und ihren Ausgleich - für die verschiedenen zu meidenden Nahrungsmittel geben.

Das Buch enthält wertvolle Hinweise über das versteckte Vorkommen von beispielsweise Milcheiweißen, Hühnereibestandteilen oder Soja in Fertignahrungsmitteln sowie über die Deklarierung von Lebensmitteln. Auch durch den Dschungel der pseudo-allergischen Reaktionen auf Nahrungsmittelbestandteile wie etwa Farbstoffe, syn- thetische Konservierungsstoffe oder Antioxidanzien und sinnvolle Maßnahmen bei Überempfindlichkeiten weiß das Werk sicher und auf das wesentliche beschränkt zu führen. Zuckermalabsorptionssyndromen wie auch der Laktoseunverträglichkeit und allgemeinen Themen wie hypoallergener Babynahrung sind eigene Kapitel gewidmet und auch pollenassoziierte Kreuzallergien sind ausreichend behandelt.

Jedem Kapitel sind umfangreiche, übersichtliche und klar verständliche Tabellen empfehlenswerter und nicht empfehlenswerter Nahrungsmittel beigefügt, die eine sehr genaue Diätplanung und -umsetzung ermöglichen.

Fazit: Die Diätempfehlungen und Lebensmittellisten sind klar ge- gliedert, umfassend und sowohl für den therapierenden Arzt wie auch für engagierte Betroffene gut nachvollziehbar. Für Patienten enthält der Band ein Glossar der wichtigsten medizinischen Fachbegriffe, so dass das Buch für Planung, Beratung und auch die Durchführung von Diäten bei Nahrungsmittelallergien und -intoleranzen sehr gut geeignet ist.

Dr. Inga Hansen, Mannheim

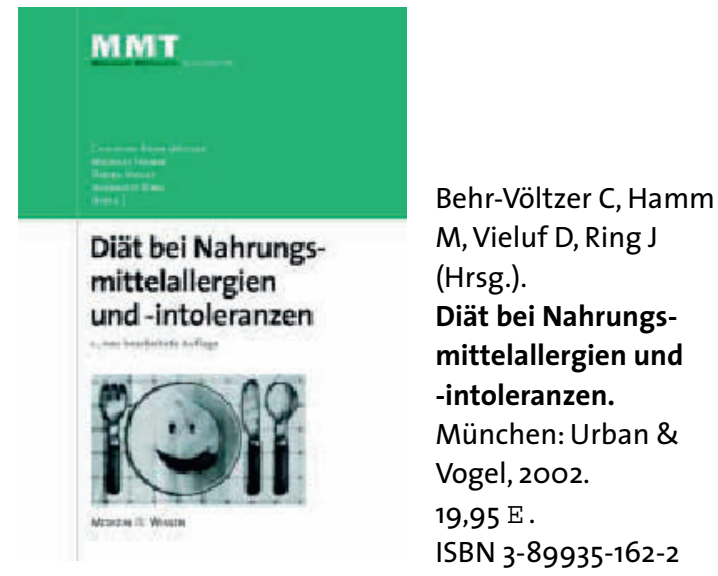

